\title{
Upregulated microRNA-214 enhances cardiac injury by targeting ITCH during coxsackievirus infection
}

\author{
ZHI-GANG CHEN, HUI LIU, JUN-BIAO ZHANG, SHAO-LI ZHANG, LI-HUA ZHAO and WAN-QIAN LIANG \\ Department of Cardiovascular Medicine, The First Affiliated Hospital of Xinxiang Medical University, \\ Weihui, Henan 453100, P.R. China
}

Received April 14,2014; Accepted January 15, 2015

DOI: $10.3892 / \mathrm{mmr} .2015 .3539$

\begin{abstract}
Viral myocarditis (VM), a severe clinical condition characterized by cardiac inflammation, is most frequently induced as a result of coxsackievirus infection. Evidence suggests that microRNAs may have significant roles in the progression of cardiac injury during coxsackievirus infection. Concurrently, microRNA (miR)-214 was found to be upregulated in the plasma and myocardial cells during this process. In the present study, eight candidate miRNAs, the functions of which are associated with myocarditis, were selected and their expression levels were evaluated by reverse transcription-quantitative polymerase chain reaction. miR-146b and miR-214 were found to have significantly upregulated expression levels in the heart tissues of patients with VM compared with those of the control subjects. Predictions via the use of online bioinformatics tools and confirmed by dual-luciferase assay and western blot analysis, revealed that ITCH, an NF- $\mathrm{\kappa B}$ signaling suppressor, was a target gene of miR-214. To investigate the biological function of miR-214, tumor necrosis factor- $\alpha$ and interleukin-6 expression levels were evaluated in HeLa cell culture supernatant. The results revealed that miR-214 overexpression enhanced the expression of the two cytokines. In addition, the function of miR-214 was partially rescued by ITCH overexpression. Subsequently, concurrent results were obtained following experiments in murine cardiac myocytes. In conclusion, the results of the present study demonstrated that miR-214 contributed to the adverse inflammatory response to viral infection of the heart during coxsackievirus infection and is therefore a potential therapeutic target for the treatment of viral myocarditis.
\end{abstract}

Correspondence to: Dr Hui Liu, Department of Cardiovascular Medicine, The First Affiliated Hospital of Xinxiang Medical University, 88 Jiankang Road, Weihui, Henan 453100, P.R. China E-mail: liuhuisunny@163.com

Key words: viral myocarditis, microRNA, coxsakievirus, ITCH

\section{Introduction}

Myocarditis is an inflammation of the heart muscle that is most often due to enteroviral infection. Coxsackievirus B3 (CVB3) is hypothesized to be the most common causative agent underlying myocarditis in humans. Viral myocarditis (VM) affects $5-20 \%$ of the human population, and is potentially fatal among infants as well as children $(1,2)$. Although VM is frequently resolved among older individuals, it may develop into chronic myocarditis and/or dilated cardiomyopathy, leading to cardiac failure (3-5). The pathogenesis of VM is based on adverse immune responses evoked by infection of the cardiac muscle with cardiotropic viruses, which leads to viral elimination as well as cardiac myocyte destruction, reparative fibrosis and heart failure (6). Due to the current dearth of effective therapies for the treatment of myocarditis, further studies of the molecular mechanisms underlying these autodestructive inflammatory signaling pathways within the immune system are required (7).

MicroRNAs (miRNAs) are short, noncoding RNA sequences that function as key gene regulators, modulating gene expression at the post-transcriptional level by targeting the 3'-untranslated region (3'UTR) of messenger RNA sequences (8). Gene expression studies have demonstrated that miRNAs are differentially expressed in heart disease, and loss-of-function studies in mice have confirmed that miRNAs are able to regulate multiple cell processes essential to heart function $(9,10)$. miRNAs have significant functions in the maintenance of normal human physiological conditions, and abnormal miRNA expression has been associated with numerous human diseases, varying from psychiatric disorders to types of malignant cancer (11-13).

Recently, a group reported that disturbed miRNA expression, including that of miR-155, -21 and $-146 \mathrm{~b}$, was associated with VM pathogenesis (10). It has also been hypothesized that miRNAs, for example miR-208b and miR-499-5p, may be used as novel biomarkers for VM diagnosis (14). In the present study, the expression levels of eight candidate miRNAs which may be involved in the pathogenesis of VM were evaluated, in order to determine which were significantly altered. Subsequently, bioinformatics tools were used to predict target genes of the identified dysregulated miRNAs, and the results were verified by dual-luciferase assay and western blot analysis. The biological functions of the identified miRNAs were also studied in mouse 
cardiac myocytes. NF- $\mathrm{kB}$ signaling was upregulated only by overexpressed miR-214, but not miR-146a. Thus, miR-214 is associated with myocardial injury during VM.

\section{Materials and methods}

Patients. All human material was obtained from the First Affiliated Hospital of Xinxiang Medical University (Weihui, China). Samples were stored at $-80^{\circ} \mathrm{C}$ immediately following collection and were available for research purposes in accordance with the Declaration of Helsinki. The study was approved by the ethics committee of The First Affiliated Hospital of Xinxiang Medical University (Weihui, China). Informed consent was obtained from the patients or their families. miRNA expression was evaluated in right ventricular septal specimens from patients with myocarditis at the acute stage $(n=8)$, within 3 months after the disease had been diagnosed, as well as a clinical history of myocarditis and confirmed viral presence in cardiac biopsies. The control subjects $(n=8)$ comprised age-matched patients presenting with unexplained ventricular tachy-arrhythmias, but with normal ejection fractions and no identified systemic or cardiac inflammation or viral presence at the time of biopsy.

Cell culture. HeLa cells were cultured in Dulbecco's modified Eagle medium supplemented with $10 \%$ fetal bovine serum (HyClone, Thermo Fisher Scientific, Waltham, MA, USA), $100 \mathrm{IU} / \mathrm{ml}$ penicillin and $10 \mathrm{mg} / \mathrm{ml}$ streptomycin. Cells were maintained at $37^{\circ} \mathrm{C}$ in a $5 \% \mathrm{CO}_{2}$ atmosphere.

Purification of neonatal murine cardiac myocytes. Six specific pathogen free BALB/c mice (8-10 weeks old) in their first day of pregnancy were were purchased from Shanghai Laboratory Animal Centre (Chinese Academy of Science, Shanghai, China). All of the mice were housed under pathogen free conditions. The mice were maintained in a controlled environment at a temperature of $25 \pm 1^{\circ} \mathrm{C}$ and relative humidity of $50-60 \%$. The environment was artificially illuminated (fluorescent lights), and the mice underwent a 12:12 h light/dark cycle and were fed a standard chow diet. The neonatal mice (postnatal 0-3 days) were sacrificed by cervical dislocation. Cardiac myocytes obtained from neonatal mice were prepared as previously described (15). Briefly, the mouse hearts were removed, minced finely and stepwise enzymatic digestion was performed using $0.25 \%$ trypsin- $0.02 \%$ EDTA. The dissociated cells were subsequently washed with complete basal Eagle's medium and the endothelial cells and fibroblasts were removed by two sequential $1 \mathrm{~h}$ adsorptions to plastic flasks at $37^{\circ} \mathrm{C}$. Non-adherent myocytes were removed, washed once, resuspended in complete basal medium and plated into tissue culture wells. Following $48 \mathrm{~h}$ of incubation, the myocytes had attached to the plastic tissue culture wells, and $>95 \%$ of the cells were identified as cardiac myocytes according to observations regarding their shape and beating activity. The cultured cells were subsequently evaluated as described below. The experiment was approved by the ethical committee of The First Affiliated Hospital of Xinxiang Medical University.

Reverse transcription-quantitative polymerase chain reaction (RT-qPCR). RT-qPCR analysis was used to examine the relative expression levels of eight candidate miRNAs. Total
RNA was extracted from the cell or tissue samples with TRIzol (Invitrogen Life Technologies, Carlsbad, CA, USA), according to the manufacturer's instructions. Eight candidate miRNAs (miR-214, miR-155, miR-146b, miR-208b, miR-21, miR-499, miR-375 and miR-125b) were selected, which had previously been reported to be associated with VM pathogenesis $(10,14,16,17)$, and their expression levels were evaluated using TaqMan miRNA RT-Real Time PCR (Applied Biosystems, Foster City, CA, USA). Single-stranded complementary DNA was synthesized using the TaqMan MicroRNA Reverse Transcription kit (Applied Biosystems) and subsequently amplified with the TaqMan Universal PCR Master mix (Applied Biosystems), alongside the miRNA-specific TaqMan MGB probes (Applied Biosystems). The reaction mixture was incubated at $95^{\circ} \mathrm{C}$ for $30 \mathrm{sec}$, followed by 40 cycles of $95^{\circ} \mathrm{C}$ for $8 \mathrm{sec}$ and $60^{\circ} \mathrm{C}$ for $30 \mathrm{sec}$. The U6 small nuclear RNA was used for normalization. Each sample, within each group, was evaluated in triplicate and each experiment was repeated $\geq 3$ times.

Target gene prediction. Potential target genes were searched for using the online bioinformatics tool TargetScan Release 6.2 (http://www.targetscan.org/) (18). The results suggested that ITCH mRNA may be directly targeted by miR-214.

Dual luciferase assay. The full length of ITCH 3'UTR was cloned into a pGL3 vector (Promega Corp., Madison, WI, USA), downstream of the firefly luciferase coding region in order to produce the luciferase reporter vector. For the luciferase reporter assays, HeLa cells were seeded into 48-well plates. miRNA mimics, miRNA antagonists or their corresponding controls and luciferase reporter vectors were co-transfected using Lipofectamine ${ }^{\circledR} 2000$ (Invitrogen Life Technologies). The pRT-TK vector, which expresses Renilla luciferase was used as a transfection control. Cells were harvested following two days of cotransfection and evaluated with the Dual-Luciferase assay (Promega Corp.). Each transfection was performed in triplicate in three independent experiments. The results are expressed as relative luciferase activity: (Firefly luciferase/Renilla luciferase). A reporter vector containing mutant ITCH 3'UTR was constructed using Fast Mutagenesis system (Beijing TransGen Biotech Co., Ltd, Beijing, China), to identify the binding region of miR-214. The binding region sequence of miR-214 (CCUGCUG) was replaced with CAUACUA. The wild type or mutant ITCH 3'UTR reporter vector was then transfected into HEK293T cells (China Infrastruture of Cell Line Resources, Beijing, China) alongside the miR-214 mimic or inhibitor using lipofectamine 2000. A total of $48 \mathrm{~h}$ post-transfection, the cells were lysed by incubation in Passive Lysis buffer on ice for $15 \mathrm{~min}$ and luciferase activity was detected using the Dual-Luciferase Assay kit.

Western blot analysis. Protein extracts were boiled in SDS/ $\beta$-mercaptoethanol sample buffer, and $20 \mu \mathrm{g}$ samples were loaded onto $8 \%$ polyacrylamide gels. The proteins were separated by electrophoresis, and blotted onto polyvinylidene difluoride membranes (Amersham Pharmacia Biotech, St. Albans, Herts, UK) via electrophoretic transfer. The membrane was incubated with rabbit polyclonal anti-human 


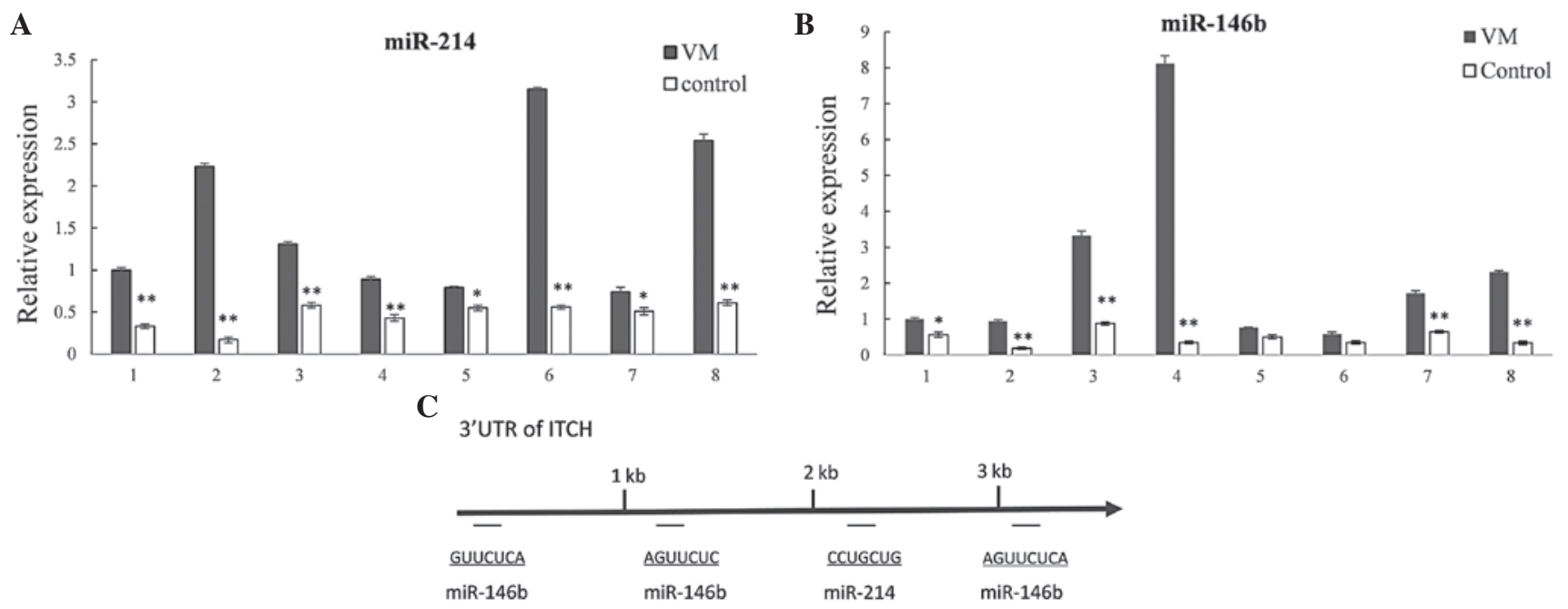

Figure 1. miR-214 and miR-146b are downregulated in the heart tissue of patients with VM. The relative expression levels of (A) miR-214 and (B) miR-146b were detected by reverse transcription-quantitative polymerase chain reaction in right ventricular septal biopsies from patients with acute myocarditis with a definite clinical history of myocarditis and confirmed virus presence in the cardiac biopsies and age-matched controls. The results were analyzed using t-test. ${ }^{*} \mathrm{P}<0.05,{ }^{* *} \mathrm{P}<0.01$ vs. VM tissue. (C) Bioinformatics prediction results indicated that ITCH may be a target gene of miR-214 and miR-146b. Three miR-146b binding regions and one miR-214 binding region were identified in the ITCH 3'UTR. miR, microRNA; VM, viral myocarditis; 3'UTR, 3' untranslated region.

A

pGL3 control vector

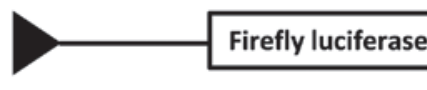
ITCH 3'UTR

B
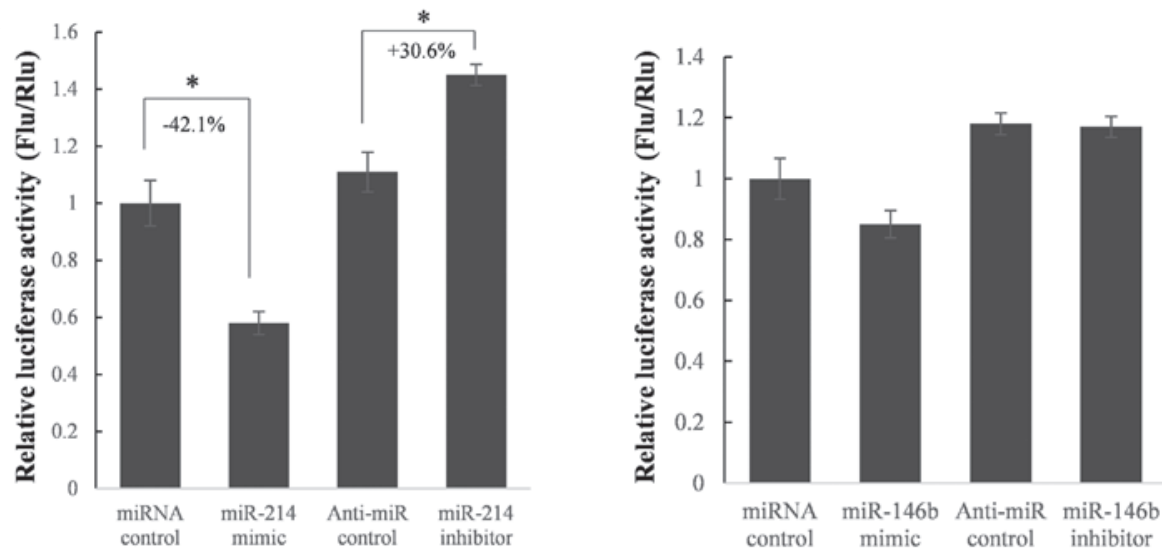

C

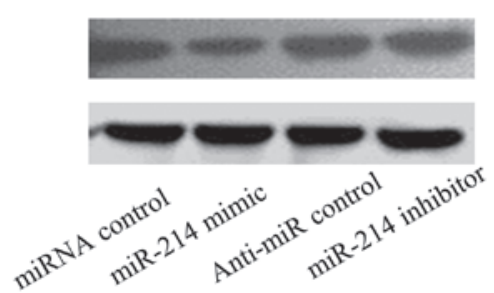

ITCH

$\beta$-actin

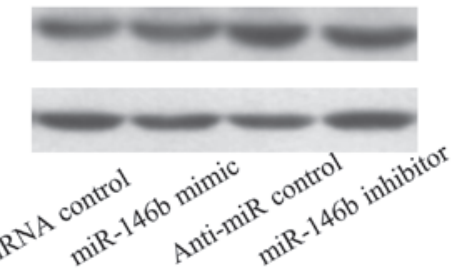

D
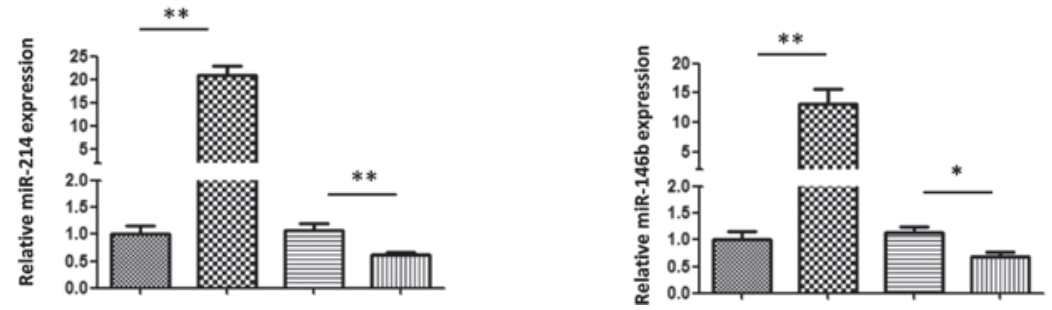

miRNA control
miR-146b mimic

Anti-miR control

Figure 2. The expression of ITCH is suppressed by miR-214, but not miR-146b, in HeLa cells. (A) Schematic diagram of the insertion of the predicted ITCH 3'UTR into the pGL3 vector. (B) Dual luciferase assay to confirm whether miR-214 targeted the 3'UTR of ITCH. HeLa cells were cotransfected with pGL3-ITCH, miR-214 or miR-146b mimic or inhibitor. The pRL-TK vector was used as a transfection control. (C) Western blot analysis was used to evaluate ITCH protein expression levels in each group. (D) miR-214 and miR-146b expression levels were examined with reverse transcription-quantitative polymerase chain reaction. The results were analyzed using t-test. ${ }^{*} \mathrm{P}<0.05,{ }^{* *} \mathrm{P}<0.01$. miR, microRNA; 3'UTR, 3 ' untranslated region. 
A

\begin{tabular}{|c|c|c|c|c|c|c|c|c|c|c|}
\hline \multirow[b]{2}{*}{ CVB3 (h) } & \multicolumn{5}{|c|}{ miRNA control } & \multicolumn{5}{|c|}{ miR-214 mimic } \\
\hline & 0 & 0.5 & 1 & 12 & 24 & 0 & 0.5 & 1 & 12 & 24 \\
\hline ITCH & & & & & & $=$ & - & - & - & $=$ \\
\hline
\end{tabular}

B
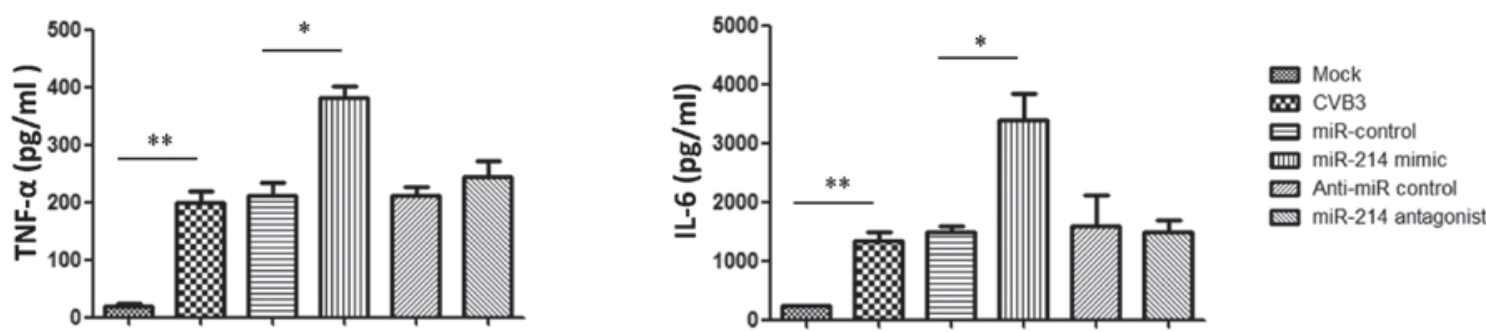

C
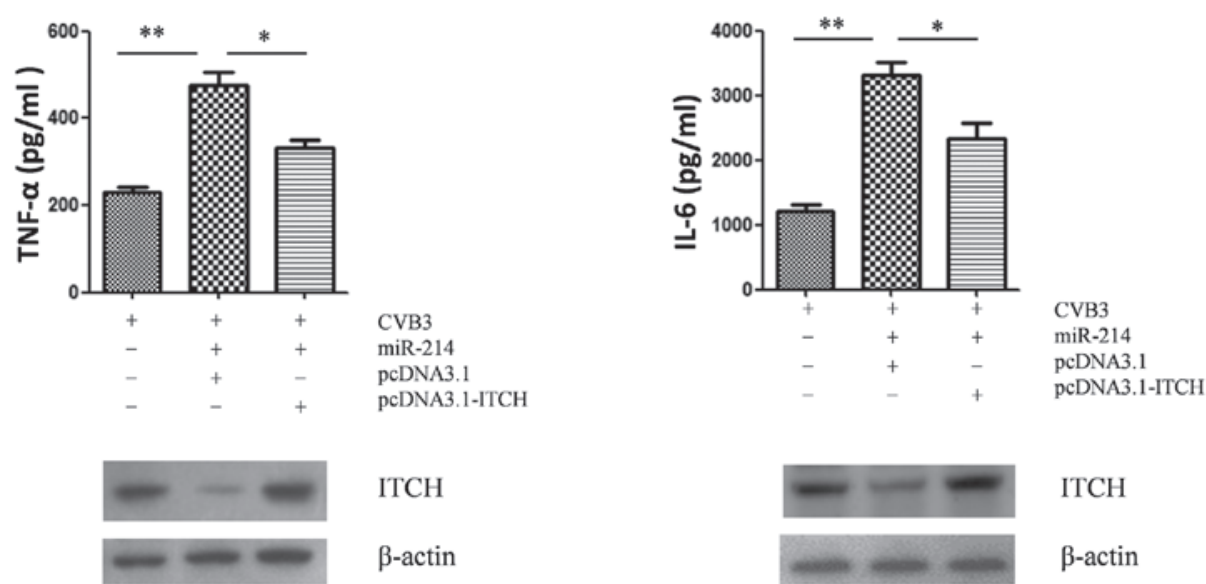

Figure 3. miR-214 upregulates TNF- $\alpha$ and IL-6 expression by targeting ITCH. (A) HeLa cells were transfected with miR-214 mimic, and $24 \mathrm{~h}$ after transfection, cells were infected with CVB3. ITCH protein levels were detected by western blot analysis at five time-points following CVB3 infection. (B) Expression levels of TNF- $\alpha$ and IL-6 in cell culture supernatants were determined by enzyme-linked immunosorbent assay. (C) Expression levels of TNF- $\alpha$ and IL-6 in cell culture supernatants were determined when HeLa cells were cotransfected with miR-214 and ITCH expression vector. Western blot analysis was used to detect ITCH protein expression. The results of paired groups were analyzed using t-test. ${ }^{*} \mathrm{P}<0.05,{ }^{* *} \mathrm{P}<0.01$. TNF- $\alpha$, tumor necrosis factor- $\alpha$; IL- 6 , interleukin-6; CVB3, coxsackievirus B3.

ITCH antibody (1:500; cat. no. ab31097; Abcam, Cambridge, MA, USA) and mouse monoclonal anti-human $\beta$-actin antibody (1:1,000; cat. no. sc-47778; Santa Cruz Biotechnology Inc., Dallas, TX, USA) for $1 \mathrm{~h}$ at $37^{\circ} \mathrm{C}$. The membrane was subsequently incubated with horseradish peroxidase-conjugated goat anti-rabbit (1:5,000; cat. no. sc-2004) or goat anti-mouse (1:10,000; cat. no. sc-2060) secondary antibodies (Santa Cruz Biotechnology, Inc.) for $1 \mathrm{~h}$ at $37^{\circ} \mathrm{C}$. Detection was performed via enhanced chemiluminescence using an ECL kit (Pierce Biotechnology Inc., Thermo Fisher Scientific, Rockford, IL, USA). $\beta$-actin was used as a loading control.

Homology analysis. The sequences of human and mouse ITCH gene were downloaded from GenBank (http://www. ncbi.nlm.nih.gov/genbank/) and analyzed using CLUSTAL X, version 1.83 (http://www.clustal.org/) (19).

Cytokine assays. The levels of tumor necrosis factor (TNF)- $\alpha$, interleukin (IL)-1 $\beta$, IL-6 and monocyte chemoattractant protein (MCP)-1 of cell culture supernatants were determined with
ELISA (eBioscience Inc., San Diego, CA, USA) according to the manufacturer's instructions.

Statistical analysis. Data were analyzed with SPSS Statistical Package version 16 (SPSS, Inc., Chicago, IL, USA). Independent two group's analyses are used t-test. $\mathrm{P}<0.05$ was considered to indicate a statistically significant difference.

\section{Results}

$m i R-214$ and $m i R-146 b$ are downregulated in the heart tissue of patients with VM. In the present study, the expression levels of eight miRNAs, which were previously reported to exhibit altered expression levels during CVB3 infection, were evaluated in heart tissue samples from patients with CVB3-infected VM. miR-214 and miR-146b were revealed to be significantly upregulated (Fig. 1A and B). Using bioinformatics tools, ITCH, an E3 ubiquitin ligase which functions as an NF- $\mathrm{KB}$ suppressor, was identified as a predicted target gene of miR-214 and miR-146b (Fig. 1C). 
A

ITCH 3'UtR WT 5'... CaUguauaugucuUC-cCUGCUGU..

miR-214 3' UGACGGACAGACACGGACGACA

MGACGGACAGACACGGACGACA

ITCH3'UTR MU 5'...CAUGUAUAUGUCUUC-CAUACUAU.

miR-214 binding site
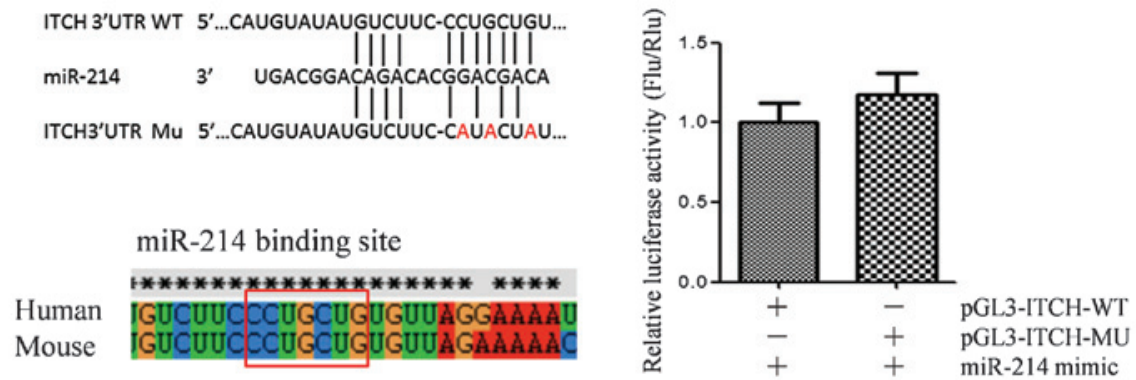

B

miRNA control

miR-214 mimic

\begin{tabular}{|c|c|c|c|c|c|c|c|c|c|}
\hline CVB3 (h) & 0.5 & 1 & 12 & 24 & 0 & 0.5 & 1 & 12 & 24 \\
\hline ITCH & - & & & & & & $=$ & - & - \\
\hline$\beta$-actin & mens & $=0$ & $=$ & & & $=$ & $=0$ & now & semen \\
\hline
\end{tabular}

C
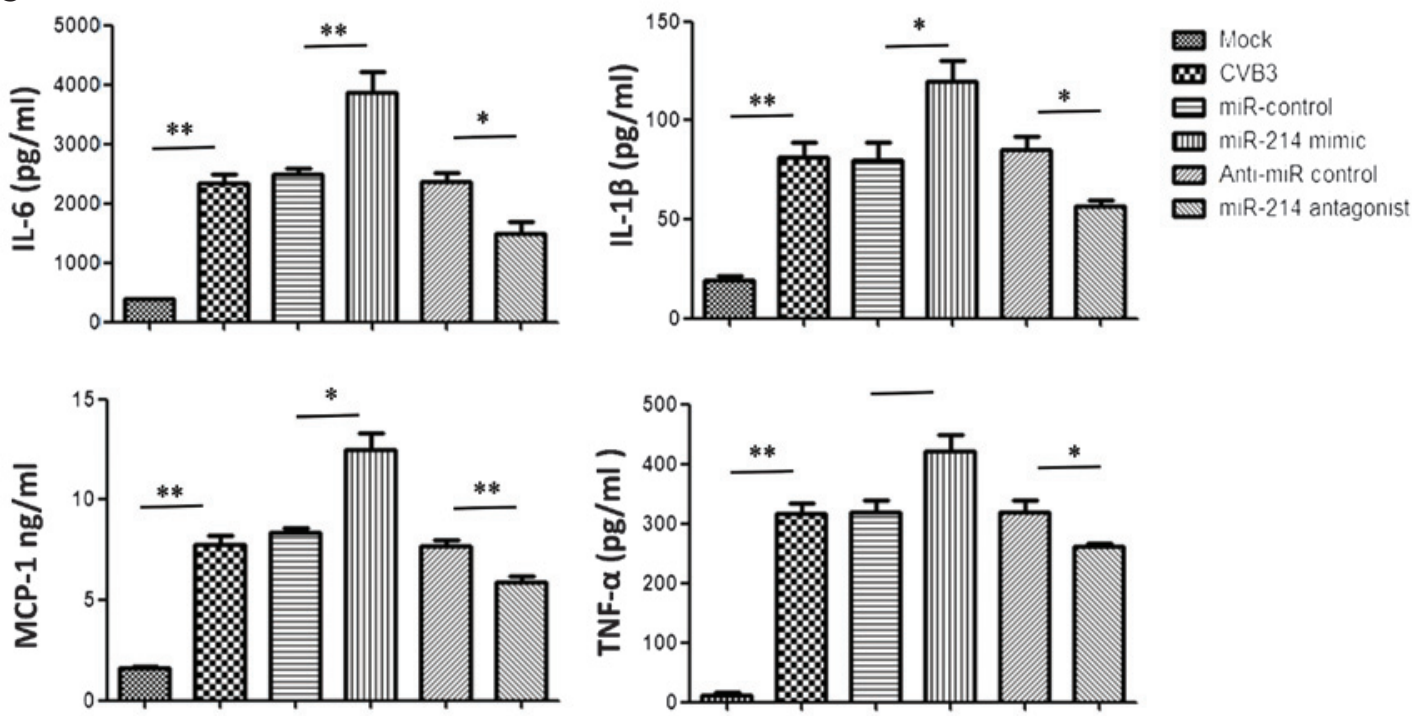

Figure 4. miR-214 upregulates TNF- $\alpha$, IL-1 $\beta$, IL-6 and MCP-1 expression by targeting ITCH in murine cardiac myocytes. (A) Homology analysis of miR-214 binding site in human and mouse ITCH 3'UTR. Dual luciferase assay was used to confirm the location of the miR-214 binding site in ITCH 3'UTR. (B) Murine cardiac myocytes were transfected with miR-214 mimic, and $24 \mathrm{~h}$ after transfection, cells were infected with CVB3. ITCH protein levels were evaluated by western blot analysis at five time-points following CVB3 infection. (C) Levels of TNF- $\alpha$, IL-1 $\beta$, IL- 6 and MCP-1 expression in cell culture supernatants were determined by enzyme-linked immunosorbent assay. The results of paired groups were analyzed using t-test. "P $<0.05,{ }^{* *} \mathrm{P}<0.01$. TNF- $\alpha$, tumor necrosis factor- $\alpha$; IL, interleukin; MCP-1, monocyte chemoattractant protein-1; 3'UTR, 3' untranslated region; CVB3, coxsackievirus B3; WT, wild-type; MU, mutant.

The expression of ITCH is suppressed by miR-214, but not miR-146b, in HeLa cells. To confirm whether the expression of ITCH was supressed by these miRNAs, the full length $(3.6 \mathrm{~kb})$ ITCH 3'UTR was cloned into a pGL3 vector downstream of the luciferase coding region (Fig. 2A). The results of a dual-luciferase assay in HeLa cells indicated that miR-214 significantly supressed firefly luciferase expression by binding to the 3'UTR of ITCH; however, miR-146b did not significantly influence luciferase expression levels (Fig. 2B). To further confirm whether miR-214 repressed ITCH expression, miR-214 was overexpressed and knocked down in HeLa cells, in which ITCH expression was detectable (Fig. 2C). Protein expression levels of ITCH were evaluated by western blot analysis (Fig. 2C), and expression levels of miR-214 and miR-146b were detected by RT-qPCR (Fig. 2D). As shown in Fig. 2B and C, ITCH expression levels were significantly reduced by the miR-214 mimic and slightly upregulated by the miR-214 inhibitor, which indicated that ITCH was a target gene of miR-214.

miR-214 upregulates TNF- $\alpha$ and IL- 6 expression by targeting $I T C H$. The NF- $\mathrm{kB}$ family of transcription factors is crucial in the mediation of inflammatory responses. ITCH is a key component of the ubiquitin-editing complex required for termination of the pro-inflammatory activation of the c-Jun $\mathrm{N}$-terminal kinases and NF- $\mathrm{kB}$. Following transfection with miR-214 mimic and CVB3 infection, ITCH expression levels were markedly downregulated, compared with those of cells transfected with the miRNA control (Fig. 3A). To further investigate the biological functions of miR-214 overexpres- 
sion, the expression levels of TNF- $\alpha$ and IL- 6 in cell culture supernatants were examined. As indicated in Fig. 3B, CVB3 infection induced upregulation of TNF- $\alpha$ and IL-6 levels, and following transfection with miR-214 mimic, the concentration of these cytokines was significantly enhanced $(\mathrm{P}<0.05)$. Following cotransfection with ITCH expression vector, the expression levels of TNF- $\alpha$ and IL-6 were significantly reduced (Fig. 3C), indicating that miR-214 promoted cell inflammatory responses partially via the suppression of ITCH expression.

miR-214 upregulates TNF- $\alpha, I L-1 \beta, I L-6$ and MCP-1 expression by targeting ITCH in murine cardiac myocytes. To further investigate this overexpression function in cardiac cells, cardiac myocytes were purified from neonatal mice. Prior to the functional study, the miR-214 binding site in ITCH 3'UTR was identified and the sequences of the human and mouse ITCH 3'UTR were aligned. As exhibited in Fig. 4A, when three nucleotides were mutated, luciferase activity was no longer repressed by miR-214, indicating that the miR-214 binding site was CCUGCUG. Furthermore, this region is highly conserved between human and mouse. Following infection with CVB3, the expression of ITCH was upregulated in a time-dependent manner in those cells transfected with the miRNA control. An analogous effect on ITCH expression was observed in those cells transfected with miR-214 mimic; however, the ITCH protein levels were markedly lower in mouse cardiac myocytes (Fig. 4B). Furthermore, the concentrations of TNF- $\alpha$, IL-6, IL-1 $\beta$ and MCP-1 in cell culture supernatants were upregulated by miR-214 mimic transfection and downregulated by miR-214 antagonist transfection (Fig. 4C).

\section{Discussion}

Myocarditis most frequently occurs as a result of infection by enteroviruses, and CVB3 is hypothesized to be the most common causative factor in human myocarditis (6). Despite decades of extensive research, the pathogenesis of VM has remained elusive, and there is currently no effective therapy available for the treatment of this disease (20). Experimental studies have revealed that although CVB3 is able to directly destroy the myocardium $(21,22)$, it is the associated aberrant inflammatory response that is primarily responsible for the induction of myocyte damage $(23,24)$. Additionally, clinical studies have identified enhanced levels of circulating TNF- $\alpha$, IL-1 $\beta$, IL-6 and other pro-inflammatory cytokines in patients with myocarditis $(25,26)$. Furthermore, specific immunosuppressive agents may be used to control the inflammatory response in clinical therapies (27). Therefore, modulation of the inflammatory response represents a potential therapeutic strategy for the treatment of viral myocarditis.

The expression of miRNAs, which function as key gene regulators, was found to be disturbed during CVB3 infection, and certain miRNAs were revealed to have promotional and/or inhibitory roles in the mediation of CVB3 infection. In the present study, eight candidate miRNAs, the functions of which are associated with myocarditis, were selected $(10,14,28,29)$, and their expression was evaluated by RT-qPCR. The results revealed that miR-146b and miR-214 expression levels were significantly upregulated in VM tissues compared with those of the control tissues. Online bioinformatics tools were used to predict ITCH, an NF- $\mathrm{NB}$ signaling suppressor, as a target gene of miR-214. This prediction was verified by dual-luciferase assay and western blot analysis. To investigate the biological function of miR-214, TNF- $\alpha$ and IL-6 expression levels were evaluated in HeLa cell culture supernatants. It was demonstrated that the expression levels of TNF- $\alpha$ and IL- 6 were upregulated by miR-214 mimic transfection. There was no significant difference in TNF- $\alpha$ or IL-6 expression between the miR-214 knockdown group and that of the control group, although this may have been a result of the low expression levels of miR-214 in HeLa cells. This hypothesis was indirectly confirmed by the experiments performed in murine cardiac myocytes, where the expression of cytokines was significantly reduced by transfection with the miR-214 antagonist.

In conclusion, to the best of our knowledge, the present study was the first to report that the upregulation of miR-214 expression was associated with VM pathogenesis by targeting ITCH. Overexpression of miR-214 upregulated the expression of cytokines and chemokines that enhance myocardial inflammation by weakening NF-кB pathway feedback signaling.

\section{References}

1. Duncan BW, Bohn DJ, Atz AM, French JW, Laussen PC and Wessel DL: Mechanical circulatory support for the treatment of children with acute fulminant myocarditis. J Thorac Cardiovasc Surg 122: 440-448, 2001.

2. Woodruff JF: Viral myocarditis. A review. Am J Pathol 101: 425-484, 1980

3. Bowles NE, Richardson PJ, Olsen EG and Archard LC: Detection of Coxsackie-B-virus-specific RNA sequences in myocardial biopsy samples from patients with myocarditis and dilated cardiomyopathy. Lancet 1: 1120-1123, 1986.

4. Kandolf R, Klingel K, Mertsching H, et al: Molecular studies on enteroviral heart disease: patterns of acute and persistent infections. Eur Heart J 12 (Suppl D): 49-55, 1991.

5. Morimoto S, Hiramitsu S, Yamada K, et al: Clinical and pathologic features of chronic myocarditis: four autopsy cases presenting as dilated cardiomyopathy in life. Am J Cardiovase Pathol 4: 181-191, 1992.

6. Maier R, Krebs P and Ludewig B: Immunopathological basis of virus-induced myocarditis. Clin Dev Immunol 11: 1-5, 2004.

7. Shauer A, Gotsman I, Keren A, et al: Acute viral myocarditis: current concepts in diagnosis and treatment. Isr Med Assoc J 15: 180-185, 2013.

8. Bartel DP: MicroRNAs: Genomics, biogenesis, mechanism, and function. Cell 116: 281-297, 2004.

9. De Rosa S, Curcio A and Indolfi C: Emerging role of microRNAs in cardiovascular diseases. Circ J 78: 567-575, 2014.

10. Corsten MF, Papageorgiou A, Verhesen W, et al: MicroRNA profiling identifies microRNA-155 as an adverse mediator of cardiac injury and dysfunction during acute viral myocarditis. Circ Res 111: 415-425, 2012.

11. Maes OC, Chertkow HM, Wang E and Schipper HM: MicroRNA: implications for alzheimer disease and other human CNS disorders. Curr Genomics 10: 154-168, 2009.

12. Xu J, Li Y, Wang F, et al: Suppressed miR-424 expression via upregulation of target gene Chk1 contributes to the progression of cervical cancer. Oncogene 32: 976-987, 2013.

13. Farazi TA, Hoell JI, Morozov P and Tuschl T: MicroRNAs in human cancer. Adv Exp Med Biol 774: 1-20, 2013.

14. Corsten MF, Dennert R, Jochems S, et al: Circulating MicroRNA-208b and MicroRNA-499 reflect myocardial damage in cardiovascular disease. Circ Cardiovasc Genet 3: 499-506, 2010.

15. Shen Y, Xu W, Chu YW, Wang Y, Liu QS and Xiong SD: Coxsackievirus group $\mathrm{B}$ type 3 infection upregulates expression of monocyte chemoattractant protein 1 in cardiac myocytes, which leads to enhanced migration of mononuclear cells in viral myocarditis. J Virol 78: 12548-12556, 2004.

16. Liu YL, Wu W, Xue Y, et al: MicroRNA-21 and $-146 \mathrm{~b}$ are involved in the pathogenesis of murine viral myocarditis by regulating TH-17 differentiation. Arch Virol 158: 1953-1963, 2013. 
17. Tijsen AJ, Pinto YM and Creemers EE: Circulating microRNAs as diagnostic biomarkers for cardiovascular diseases. Am J Physiol Heart Circ Physiol 303: H1085-H1095, 2012.

18. Lewis BP, Burge CB and Bartel DP: Conserved seed pairing, often flanked by adenosines, indicates that thousands of human genes are microRNA targets. Cell 120: 15-20, 2005.

19. Thompson JD, Gibson TJ, Plewniak F, Jeanmougin F and Higgins DG: The CLUSTAL_X windows interface: Flexible strategies for multiple sequence alignment aided by quality analysis tools. Nucleic Acids Res 25: 4876-4882, 1997.

20. Esfandiarei M and McManus BM: Molecular biology and pathogenesis of viral myocarditis. Annu Rev Pathol 3: 127-155, 2008.

21. Klingel $\mathrm{K}$ and Kandolf R: The role of enterovirus replication in the development of acute and chronic heart muscle disease in different immunocompetent mouse strains. Scand J Infect Dis Suppl 88: 79-85, 1993.

22. Fuse K, Chan G,Liu Y, et al: Myeloid differentiation factor-88 plays a crucial role in the pathogenesis of Coxsackievirus B3-induced myocarditis and influences type I interferon production. Circulation 112: 2276-2285, 2005 .
23. Leipner C, Grün K, Borchers M and Stelzner A: The outcome of coxsackievirus B3-(CVB3-) induced myocarditis is influenced by the cellular immune status. Herz 25: 245-248, 2000.

24. Calabrese F and Thiene G: Myocarditis and inflammatory cardiomyopathy: microbiological and molecular biological aspects. Cardiovasc Res 60: 11-25, 2003.

25. Matsumori A, Yamada T, Suzuki H, Matoba Y and Sasayama S: Increased circulating cytokines in patients with myocarditis and cardiomyopathy. Br Heart J 72: 561-566, 1994.

26. Levine B, Kalman J, Mayer L, Fillit HM and Packer M: Elevated circulating levels of tumor necrosis factor in severe chronic heart failure. N Engl J Med 323: 236-241, 1990.

27. Schultz JC, Hilliard AA, Cooper LT Jr and Rihal CS: Diagnosis and treatment of viral myocarditis. Mayo Clin Proc 84: 1001-1009, 2009.

28. Xu HF, Ding YJ, Shen YW, et al: MicroRNA-1 represses Cx43 expression in viral myocarditis. Mol Cell Biochem 362: 141-148, 2012.

29. Hemida MG, Ye X, Zhang HM, et al: MicroRNA-203 enhances coxsackievirus B3 replication through targeting zinc finger protein-148. Cell Mol Life Sci 70: 277-291, 2013. 